\title{
CLASSIFYING PL 5-MANIFOLDS UP TO REGULAR GENUS SEVEN
}

\author{
MARIA RITA CASALI AND CARLO GAGLIARDI
}

(Communicated by James West)

\begin{abstract}
In the present paper, we show that the only closed orientable 5manifolds of regular genus less or equal than seven are the 5-sphere $\mathbb{S}^{5}$ and the connected sums of $m$ copies of $\mathbb{S}^{1} \times \mathbb{S}^{4}$, with $m \leq 7$. As a consequence, the genus of $\mathbb{S}^{3} \times \mathbb{S}^{2}$ is proved to be eight. This suggests a possible approach to the (3-dimensional) Poincare Conjecture, via the well-known classification of simply connected 5-manifolds, obtained by Smale and Barden.
\end{abstract}

\section{Statements}

The possibility of representing a large class of polyhedra, including all PLmanifolds of dimension $n$, by means of $(n+1)$-coloured graphs is well known (see, as general references, [FGG, BM, V]).

Within this representation theory, a nonnegative combinatorial invariant $\mathscr{G}\left(M^{n}\right)$, called the regular genus of $M^{n}$, has been introduced for each $n$ manifold $M^{n}$. It extends to dimension $n$ the classical notions of genus of a surface and of Heegaard genus of a 3-manifold [G2]; it further characterizes the $n$-sphere $\mathbb{S}^{n}$ among all closed $n$-manifolds, as follows [FG]:

$$
\mathscr{G}\left(M^{n}\right)=0 \quad \text { iff } \quad M^{n} \cong \mathbb{S}^{n}
$$

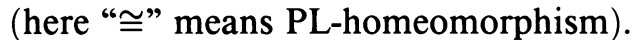

In the present paper, we obtain the classification of all closed connected orientable 5-dimensional PL-manifolds with regular genus less than or equal to 7:

Main Theorem. Let $M^{5}$ be a closed connected orientable PL 5-manifold. Then

$$
1 \leq \mathscr{G}\left(M^{5}\right)=m \leq 7 \quad \text { iff } \quad M^{5} \cong \underset{m}{\#}\left(\mathbb{S}^{1} \times \mathbb{S}^{4}\right)
$$

(where $\#_{m}\left(\mathbb{S}^{1} \times \mathbb{S}^{4}\right)$ denotes the connected sum of $m$ copies of $\left.\mathbb{S}^{1} \times \mathbb{S}^{4}\right)$.

As a direct consequence, we have

Received by the editors May 10,1991; the contents of this paper have been presented by the first author to XIV Congresso U.M.I., Catania, Italy, September 19-25, 1991.

1991 Mathematics Subject Classification. Primary 57N15, 57Q15; Secondary 05C10.

Key words and phrases. 5-Manifolds, regular genus, classification, products of spheres and manifolds, handlebodies, Poincaré Conjecture, edge-coloured graphs, 2-cell embeddings.

This work was performed under the auspicies of the G.N.S.A.G.A.-C.N.R., and within the Project "Geometria Reale e Complessa", supported by M.U.R.S.T. of Italy. 
Corollary I. (a) $\mathscr{G}\left(\mathbb{S}^{3} \times \mathbb{S}^{2}\right)=8$.

(b) For every closed orientable PL 3-manifold $M^{3}, \mathscr{G}\left(M^{3} \times \mathbb{S}^{2}\right) \geq 8$.

The proof of the above results will be presented in $\S 3$.

Remark A. By [B], if $\Sigma^{3}$ is a homotopy 3-sphere, then $\Sigma^{3} \times \mathbb{S}^{2} \cong \mathbb{S}^{3} \times \mathbb{S}^{2}$; therefore, by Corollary I(a), $\mathscr{G}\left(\Sigma^{3} \times \mathbb{S}^{2}\right)=8$. We believe that the above relation characterizes homotopy 3-spheres among orientable 3-manifolds (i.e., that $\mathscr{G}\left(M^{3} \times \mathbb{S}^{2}\right)=8$ iff $M^{3}$ is a homotopy 3-sphere). On the other hand, since Corollary I gives a positive answer to parts $(a)$ and $\left(b^{\prime}\right)$ of Conjecture I of [GG] (see also [G3]), we can state the following stronger

Conjecture $\mathrm{I}^{\prime}$. Let $M^{3}$ be any closed orientable 3-manifold. Then

$$
\mathscr{G}\left(M^{3} \times \mathbb{S}^{2}\right)=8 \quad \text { iff } M^{3} \cong \mathbb{S}^{3} \text {. }
$$

Conjecture $I^{\prime}$ seems, of course, of hard solution: in fact, we recall that it actually implies the (3-dimensional) Poincaré Conjecture, by the classification of simply connected 5-manifolds, obtained in [S, B]. Let us propose the following general problem about the genus of product manifolds, presented as a sequence of conjectures, for the sake of conciseness.

Conjecture $\mathrm{II}_{n}$. For every closed connected orientable 3-manifold $M^{3}$, $\mathscr{G}\left(M^{3} \times \mathbb{S}^{n}\right) \geq \mathscr{G}\left(M^{3} \times \mathbb{D}^{n}\right)$ (where $\mathbb{D}^{n}$ denotes the $n$-disk).

Remark B. Conjectures $\mathrm{II}_{1}$ and $\mathrm{II}_{2}$ together imply the Poincaré Conjecture for all closed 3-manifolds $M^{3}$ of Heegaard genus $\mathscr{H}\left(M^{3}\right) \geq 5$.

In fact, by [CP], $\mathscr{G}\left(M^{h}\right) \geq \mathscr{G}\left(\partial M^{h}\right)$, where $M^{h}$ is any $h$-manifold with boundary $\partial M^{h}$ and $\mathscr{G}\left(\partial M^{h}\right)$ denotes the sum of the genera of its components. Hence, assuming Conjecture $\mathrm{II}_{2}, \mathscr{G}\left(M^{3} \times \mathbb{S}^{2}\right) \geq \mathscr{G}\left(M^{3} \times \mathbb{D}^{2}\right) \geq \mathscr{G}\left(M^{3} \times \mathbb{S}^{1}\right)$; furthermore, assuming Conjecture $\mathrm{II}_{1}, \mathscr{G}\left(M^{3} \times \mathbb{S}^{1}\right) \geq \mathscr{G}\left(M^{3} \times \mathbb{D}^{1}\right) \geq 2 \mathscr{G}\left(M^{3}\right)=$ $2 \mathscr{H}\left(M^{3}\right)$.

This proves that, if $\mathscr{H}\left(M^{3}\right) \geq 5$, then $\mathscr{G}\left(M^{3} \times \mathbb{S}^{2}\right) \geq 2 \mathscr{H}\left(M^{3}\right) \geq 10$, and therefore $M^{3}$ is not a homotopy sphere, since for each homotopy 3-sphere $\Sigma^{3}$ we have $\mathscr{G}\left(\Sigma^{3} \times \mathbb{S}^{2}\right)=\mathscr{G}\left(\mathbb{S}^{3} \times \mathbb{S}^{2}\right)=8$, by Corollary I.

\section{Preliminaries AND NOtations}

In this paper, we shall work with manifolds (closed, connected, and orientable, unless otherwise stated) and maps in the PL-category, for which we refer to [Gl] or [RS]; we will often identify a ball-complex $K$ with its associated polyhedron and every homeomorphic space. For graph theory, see [W].

An $(n+1)$-coloured graph is a pair $(\Gamma, \gamma)$, where $\Gamma=(V(\Gamma), E(\Gamma))$ is a multigraph (i.e., multiple edges are allowed, but loops are forbidden), regular of degree $n+1$, and $\gamma: E(\Gamma) \rightarrow \Delta_{n}=\{i \in \mathbb{Z} \mid 0 \leq i \leq n\}$ is a proper edge-colouring on $\Gamma$ (i.e., $\gamma(\mathbf{e}) \neq \gamma(\mathbf{f})$ for any two adjacent edges $\mathbf{e}, \mathbf{f} \in E(\Gamma)$. For the sake of conciseness, we shall often denote the $(n+1)$-coloured graph $(\Gamma, \gamma)$ simply by the symbol $\Gamma$ of its underlying multigraph.

For each $\mathscr{B} \subseteq \Delta_{n}$, set $\Gamma_{\mathscr{B}}=\left(V(\Gamma), \gamma^{-1}(\mathscr{B})\right)$; each connected component of $\Gamma_{\mathscr{B}}$ is called a $\mathscr{B}$-residue, or an $m$-residue if the cardinality \# $\mathscr{B}$ of $\mathscr{B}$ is $m$. For each $i \in \Delta_{n}$, set $\hat{i}=\Delta_{n}-\{i\}$. The symbol $\mathbf{g}(\Gamma)\left(\operatorname{resp}\right.$. $\left.\mathbf{g}_{\mathscr{B}}=\mathbf{g}_{\mathscr{B}}(\Gamma)\right)$ will denote the number of components of $\Gamma$ (resp. of $\mathscr{B}$-residues of $\Gamma$ ); if $\mathscr{B}=\{i, j\} \quad($ resp. $\mathscr{B}=\{i, j, k\})$, we shall write $\mathbf{g}_{i j}$ (resp. $\left.\mathbf{g}_{i j k}\right)$ instead of $\mathbf{g}_{\mathscr{B}}$ and $\mathbf{g}_{\hat{i} \hat{j}}$ (resp. $\mathbf{g}_{\hat{i} \hat{j} \hat{k}}$ ) instead of $\mathbf{g}_{\Delta_{n}-B}$. 
An $n$-dimensional labelled pseudocomplex $K(\Gamma)$ (see [HW, BM]) can be associated to every $(n+1)$-coloured graph $(\Gamma, \gamma)$; the construction, described in [FGG], works as follows:

(i) for each $\mathbf{v} \in V(\Gamma)$, take an $n$-simplex $\sigma(v)$, with its vertices labelled $0,1, \ldots, n$

(ii) for each $\mathbf{e} \in E(\Gamma)$ with end points $\mathbf{v}, \mathbf{w} \in V(\Gamma)$ and colour $\gamma(\mathbf{e})=i$, identify the $(n-1)$-faces of $\sigma(v)$ and $\sigma(w)$ opposite of the vertex labelled $i$.

We say that $(\Gamma, \gamma)$ represents $K(\Gamma)$ and every homeomorphic polyhedron. It is easy to check that, for each $\mathscr{B} \in \Delta_{n}$, with $\# \mathscr{B}=h \leq n$, there is a bijection between the set of $\mathscr{B}$-residues of $\Gamma$ and the set of $(n-h)$-simplices of $K(\Gamma)$, whose vertices are labelled $\Delta_{n}-\mathscr{B}$. Moreover, it is known that:

- $K(\Gamma)$ is orientable iff $\Gamma$ is a bipartite graph;

- $K(\Gamma)$ is a manifold iff, for every $n$-residue $\boldsymbol{\Theta}$ of $\Gamma, K(\boldsymbol{\theta})$ is homeomorphic to the $(n-1)$-sphere $\mathbb{S}^{n-1}$.

A crystallization of a closed connected $n$-manifold $M^{n}$ is any $(n+1)$ coloured graph $(\Gamma, \gamma)$ representing $M^{n}$, such that $\Gamma_{\hat{c}}$ is connected for each colour $c \in \Delta_{n}$. The basic results of [P1, P2] ensure the existence of crystallizations for every closed $n$-manifold. An alternative proof is contained in [LM].

In [G2] the existence is proved, for each connected bipartite $(n+1)$-coloured graph $(\Gamma, \gamma)$ of order $\# V(\Gamma)=\mathbf{p}$ and for each cyclic permutation $\varepsilon=\left(\varepsilon_{0}\right.$, $\varepsilon_{1}, \ldots, \varepsilon_{n}$ ) of $\Delta_{n}$, of a particular 2-cell embedding (see [W]) $l: \Gamma \rightarrow F_{\varepsilon}$, where $F_{\varepsilon}$ denotes the orientable closed surface of genus $\rho\left(F_{\varepsilon}\right)=\rho_{\varepsilon}(\Gamma)=1-\chi_{\varepsilon}(\Gamma) / 2$, where

$$
\chi_{\varepsilon}(\Gamma)=\sum_{c \in \mathbb{Z}_{n+1}} \mathbf{g}_{\varepsilon_{\varepsilon} \varepsilon_{c+1}}+(1-n) \mathbf{p} / 2 .
$$

The nonnegative integers $\rho(\Gamma)=\min _{\varepsilon}\left\{\rho_{\varepsilon}(\Gamma)\right\}$ and $\mathscr{G}\left(M^{n}\right)=\min \{\rho(\Gamma) \mid \Gamma$ is a crystallization of $\left.M^{n}\right\}$ are respectively called the regular genus of the graph $\Gamma$ and of the manifold $M^{n}$.

From now on, $(\Gamma, \gamma)$ is assumed to be a bipartite $(n+1)$-coloured graph representing a (possibly disconnected) closed orientable $n$-manifold. If $\Gamma$ (resp. $\Gamma_{\mathscr{B}}$, with $\left.\mathscr{B} \subseteq \Delta_{n}\right)$ has $\mathbf{g}=\mathbf{g}(\Gamma) \geq 1$ (resp. $\mathbf{g}_{\mathscr{B}}=\mathbf{g}_{\mathscr{B}}(\Gamma) \geq 1$ ) connected components $\Gamma^{(1)}, \Gamma^{(2)}, \ldots, \Gamma^{(\mathbf{g})}\left(\right.$ resp. $\left.\Xi^{(1)}, \Xi^{(2)}, \ldots, \Xi^{\left(\mathbf{g}_{B}\right)}\right)$, we define $\rho=\rho_{\varepsilon}(\Gamma)=\sum_{r} \rho_{\varepsilon}\left(\Gamma^{(r)}\right) \quad\left(\right.$ resp. $\left.\rho_{\mathscr{B}}=\rho_{\varepsilon^{\prime}}\left(\Gamma_{\mathscr{B}}\right)=\sum_{r} \rho_{\mathcal{\varepsilon}^{\prime}}\left(\Xi^{(r)}\right)\right)$, where $\rho_{\varepsilon}\left(\Gamma^{(r)}\right)$ (resp. $\rho_{\varepsilon^{\prime}}\left(\Xi^{(r)}\right)$ ) denotes the genus of $\Gamma^{(r)}$ (resp. of $\Xi^{(r)}$ ), with respect to the cyclic permutation $\varepsilon=\left(\varepsilon_{0}, \varepsilon_{1}, \ldots, \varepsilon_{n}\right)$ of $\Delta_{n}$ (resp. to the cyclic permutation $\varepsilon^{\prime}$ induced by $\varepsilon$ on the subset $\mathscr{B}$ of $\Delta_{n}$ ). For the sake of notational simplicity, we shall always write " $i$ " instead of " $\varepsilon_{i}$ ", for all $i \in \Delta_{n}$. Hence, the following formulae, written for the fundamental cyclic permutation $(0,1, \ldots, n)$, actually hold for every cyclic permutation $\left(\varepsilon_{0}, \varepsilon_{1}, \ldots, \varepsilon_{n}\right)$ of $\Delta_{n}$.

Now, the relation $\chi_{\varepsilon}(\Gamma)=2 \mathbf{g}(\Gamma)-2 \rho_{\varepsilon}(\Gamma)=2 \mathbf{g}-2 \rho$ gives

$$
\sum_{c \in \mathbb{Z}_{n+1}} \mathbf{g}_{c, c+1}+(1-n) \mathbf{p} / 2=2 \mathbf{g}-2 \rho .
$$

For each subgraph $\Gamma_{\hat{i}}$, with $i \in \Delta_{n}$, the following analogous formula holds:

$$
\mathbf{g}_{i-1, i+1}+\sum_{c \in \mathbb{Z}_{n+1}^{(i)}} \mathbf{g}_{c, c+1}+(2-n) \mathbf{p} / 2=2 \mathbf{g}_{\hat{i}}-2 \rho_{\hat{i}},
$$


where $\mathbb{Z}_{n+1}^{(i)}$ denotes the set $\Delta_{n}-\{i-1, i\}$ and the sum is $\bmod n+1$.

By substituting $\left(1_{i}\right)$ into $(1)$ and by making use of the relation

$$
2 \mathbf{g}_{i j k}=\mathbf{g}_{i j}+\mathbf{g}_{j k}+\mathbf{g}_{k i}-\mathbf{p} / 2
$$

(which is a direct consequence of $\chi(K(\Theta))=\chi\left(\mathbb{S}^{2}\right)=2$, for each $\{i, j, k\}$ residue $\Theta$ ), we obtain (for details, see [GG, Lemmas 6.1 and 6.2])

$$
\mathbf{g}_{i-1, i+1}=\mathbf{g}_{i-1, i, i+1}+\rho-\rho_{\hat{i}}+\mathbf{g}_{\hat{i}}-\mathbf{g} \text {. }
$$

The following formula is simply obtained by taking the difference between relation $\left(3_{i}\right)$, applied to the graph $(\Gamma, \gamma)$, and the same relation, applied to the partial graph $\Gamma_{\hat{j}}$, with $j \neq i \pm 1$ :

$$
\mathbf{g}_{\hat{i} \hat{j}}=\mathbf{g}_{\hat{i}}+\mathbf{g}_{\hat{j}}-\mathbf{g}+\rho-\rho_{\hat{i}}-\rho_{\hat{j}}+\rho_{\hat{i} \hat{j}} \quad(j \neq i \pm 1)
$$

where we have set $\rho_{\hat{i} \hat{j}}=\rho_{\Delta_{n}-\{i, j\}}$.

Remark C. Since $\mathbf{g}-\mathbf{g}_{\hat{i}} \geq \mathbf{g}_{\hat{j}}-\mathbf{g}_{\hat{i} \hat{j}}$ (as it is easy to check), formula (4) implies that, for every $i, j$ nonconsecutive in $\varepsilon, \rho-\rho_{\hat{i}} \geq \rho_{\hat{j}}-\rho_{\hat{i} \hat{j}}$.

Let us now restrict our attention to the case of $(\Gamma, \gamma)$ being a crystallization of a closed connected orientable 5-manifold $M^{5}$. So, $\mathbf{g}=\mathbf{g}_{\hat{c}}=1$ for every $c \in \Delta_{5}$; moreover, if we set $\rho_{\hat{i} \hat{j} \hat{k}}=\rho_{\Delta_{n}-\{i, j, k\}}$, then $\rho_{\hat{i} \hat{j} \hat{k}}=0$ for every $i, j$, $k \in \Delta_{5}$, since each 3-residue of $\Gamma$ represents a 2-sphere. Hence, relation (4), applied to the graph $\Gamma$ and to the subgroup $\Gamma_{\hat{k}}$ respectively, gives

$$
\mathbf{g}_{\hat{i} \hat{j}}=1+\rho-\rho_{\hat{i}}-\rho_{\hat{j}}+\rho_{\hat{i} \hat{j}}, \quad \text { for every } i, j \text { nonconsecutive in } \varepsilon
$$

and

(6) $\mathbf{g}_{\hat{i} \hat{j} \hat{k}}=\mathbf{g}_{\hat{i} \hat{k}}+\mathbf{g}_{\hat{j} \hat{k}}-1+\rho_{\hat{k}}-\rho_{\hat{i} \hat{k}}-\rho_{\hat{j} \hat{k}}$,

for every $i, j$ nonconsecutive in the cyclic permutation $\varepsilon_{\hat{k}}$ induced by $\varepsilon$ on $\Delta_{5}-\{k\}$.

Remark D. Since, as in Remark C, $\mathbf{g}_{\hat{k}}-\mathbf{g}_{\hat{i} \hat{k}}=1-\mathbf{g}_{\hat{i} \hat{k}} \geq \mathbf{g}_{\hat{j} \hat{k}}-\mathbf{g}_{\hat{i} \hat{j} \hat{k}}$, formula (6) actually implies that, for every $i, j$ nonconsecutive in $\varepsilon_{\hat{k}}, \rho_{\hat{k}}-\rho_{\hat{i} \hat{k}}-\rho_{\hat{j} \hat{k}} \geq 0$.

By adding formulae $\left(3_{i}\right)$ for $i \in \Delta_{5}, i$ even (resp. $i$ odd), and by making use of relation (2), the following formula (7) (resp. (8)) is obtained:

$$
\begin{aligned}
& \mathbf{g}_{\hat{0} \hat{\hat{2}} \hat{4}}=1+2 \rho-\left(\rho_{\hat{0}}+\rho_{\hat{2}}+\rho_{\hat{4}}\right), \\
& \mathbf{g}_{\hat{1} \hat{3} \hat{j}}=1+2 \rho-\left(\rho_{\hat{1}}+\rho_{\hat{3}}+\rho_{\hat{\zeta}}\right) .
\end{aligned}
$$

Moreover, since the subgraph $\Gamma_{\hat{i}}$ represents a four-dimensional sphere, the direct computation of $\chi\left(K\left(\Gamma_{\hat{i}}\right)\right)$ by means of formulae $(1),\left(1_{i}\right)$, and (2) gives

$$
\rho_{\hat{i}}=\left(\sum_{j \neq i} \rho_{\hat{i} \hat{j}}\right) / 2
$$

(see [GG, Proposition 6.3] for a detailed proof, in a more general setting).

\section{Classifying 5-Manifolds of genus $\leq 7$}

In the present section, $(\Gamma, \gamma)$ is assumed to be a crystallization of a closed connected orientable 5-manifold $M^{5}$. If $K=K(\Gamma)$ denotes the triangulation of 
$M^{5}$ associated to $\Gamma$ and $\left\{v_{c} \mid c \in \Delta_{5}\right\}$ is the vertex-set of $K$, then we may suppose that $v_{c}$ corresponds to $\Gamma_{\hat{c}}$, for each colour $c \in \Delta_{5}$. For each subset $\{i, j\}$ (resp. $\{i, j, k\})$ of distinct elements of $\Delta_{5}$, let $K(i, j)$ (resp. $K(i, j, k)$ ) be the subcomplex of $K$ generated by the vertices $v_{i}$ and $v_{j}$ (resp. $v_{i}, v_{j}$, and $v_{k}$ ), and let $K(\hat{i}, \hat{j})$ (resp. $\left.K(\hat{i}, \hat{j}, \hat{k})\right)$ be the subcomplex of $K$ generated by the vertex-set $\left\{v_{c} \mid c \in \Delta_{5}-\{i, j\}\right\}$ (resp. $\left.\left\{v_{d} \mid d \in \Delta_{5}-\{i, j, k\}\right\}\right)$. Let further $N(i, j), N(i, j, k), N(\hat{i}, \hat{j})$, and $N(\hat{i}, \hat{j}, \hat{k})$ be the regular neighbourhood in $K$ of $K(i, j), K(i, j, k), K(\hat{i}, \hat{j})$, and $K(\hat{i}, \hat{j}, \hat{k})$ respectively.

The following general results are needed in the proofs of the Main Theorem and of Corollary 1.

Lemma 1a [C]. Let $H$ be a two-dimensional pseudocomplex which contains exactly three vertices $\mathbf{a}, \mathbf{b}, \mathbf{c}$. For each pair $\mathbf{v}, \mathbf{w}$ of distinct vertices of $\mathrm{H}$, let $H(\mathbf{v}, \mathbf{w})$ be the subcomplex of $H$, generated by the vertex-set $\{\mathbf{v}, \mathbf{w}\}$. Let further [v, w] (resp. [a, b, c]) denote the number of edges of $H(\mathbf{v}, \mathbf{w})$ (resp. the number of triangles of $H$ ). If $H$ satisfies the following properties:

(i) $1 \leq[\mathbf{a}, \mathbf{c}] \leq[\mathbf{a}, \mathbf{b}]-1$;

(ii) $[\mathbf{a}, \mathbf{b}, \mathbf{c}]=[\mathbf{a}, \mathbf{b}]+[\mathbf{a}, \mathbf{c}]-1$;

(iii) the inclusion $j: H(\mathbf{b}, \mathbf{c}) \rightarrow H$ induces an epimorphism $j_{*}: \pi_{1}(H(\mathbf{b}, \mathbf{c}))$ $\rightarrow \pi_{1}(H)$,

then $H$ collapses to $H(\mathbf{b}, \mathbf{c})$.

Proof. First of all, we note that property (ii) implies the following property:

(iv) all edges of $H(\mathbf{a}, \mathbf{b})$ are faces of at least one triangle of $H$.

In fact, if an edge $\mathbf{e}$ of $H(\mathbf{a}, \mathbf{b})$ is not a face of any triangle of $H$, then any edge-loop in $H$ containing e could not be homotopic to an edge-loop of $H(\mathbf{b}, \mathbf{c})$; this obviously contradicts (iii), and hence (iv) holds, too. As a consequence of properties (i), (ii), (iv), only $r \leq[\mathbf{a}, \mathbf{c}]-1 \leq[\mathbf{a}, \mathbf{b}]-2$ edges $(\mathbf{a}, \mathbf{b})$ are faces of more than one triangle of $H$; then, some triangles of $H$ collapse from their edges " $(\mathbf{a}, \mathbf{b})$ " on their edges " $(\mathbf{a}, \mathbf{c})$ " and " $(\mathbf{b}, \mathbf{c})$ ". Hence, $H$ collapses to a subcomplex $H^{\prime}$ such that $H^{\prime}(\mathbf{b}, \mathbf{c})=H(\mathbf{b}, \mathbf{c})$. If $r=0$, it is easy to see that $[\mathbf{a}, \mathbf{c}]$ must be equal to 1 ; that is, $H^{\prime}$ is a graph collapsing to $H^{\prime}(\mathbf{b}, \mathbf{c})$. On the other hand, if $r \geq 1$, then $H^{\prime}$ satisfies again the properties (i), (ii), and (iii), with the vertices b and c exchanged of meaning. Thus, the process may be iterated, until $r=0$, leaving $H(\mathbf{b}, \mathbf{c})$ fixed.

Lemma 1b. Let $H$ be a two-dimensional pseudocomplex which contains exactly three vertices $\mathbf{a}, \mathbf{b}$, c. If $H$ satisfies the following properties:

(i) $[\mathbf{a}, \mathbf{b}]=[\mathbf{a}, \mathbf{c}]=3$;

(ii) $[\mathbf{a}, \mathbf{b}, \mathbf{c}]=[\mathbf{b}, \mathbf{c}]+3$;

(iii) for $\mathbf{z} \in\{\mathbf{b}, \mathbf{c}\}$, the inclusion $j_{\mathbf{z}}: H(\mathbf{a}, \mathbf{z}) \rightarrow H$ induces an epimorphism $j_{\mathbf{z}^{*}}: \pi_{1}(\dot{H}(\mathbf{a}, \mathbf{z})) \rightarrow \pi_{1}(H)$;

(iv) $H_{1}(H ; \mathbb{Z})$ is free and $H_{2}(H ; \mathbb{Z})=0$,

then $H$ collapses to a graph.

Proof. First of all we note, as in the proof of Lemma 1a, that property (iii) implies the following property, for every pair $\alpha, \beta$ of vertices of $H$ :

$\left(\mathrm{v}_{\boldsymbol{\alpha} \beta}\right)$ all edges of $H(\boldsymbol{\alpha}, \boldsymbol{\beta})$ are faces of at least one triangle of $H$.

Let now $\mathbf{e}_{1}, \ldots, \mathbf{e}_{h}(1 \leq h \leq 3)$ be the $h$ edges of $H(\mathbf{b}, \mathbf{c})$, which are faces of at least two triangles of $H$, and let $T_{1}, \ldots, T_{3+h}$ be the $3+h$ triangles which 
have an edge $\mathbf{e}_{r}$ as face, for $r=1, \ldots, h$. By property $\left(\mathbf{v}_{\mathbf{b}, \mathbf{c}}\right), H$ collapses to the subcomplex $H^{\prime}=H(\mathbf{a}, \mathbf{b}) \cup H(\mathbf{a}, \mathbf{c}) \cup\left\{\mathbf{e}_{1}, \ldots, \mathbf{e}_{h}\right\} \cup\left\{T_{1}, \ldots, T_{3+h}\right\}$, with $1 \leq h \leq 3$, which of course satisfies all conditions (i), (ii), (iii), (iv) and, therefore, also $\left(\mathbf{v}_{\boldsymbol{\alpha} \boldsymbol{\beta}}\right)$, for every $\boldsymbol{\alpha}, \boldsymbol{\beta} \in\{\mathbf{a}, \mathbf{b}, \mathbf{c}\}$. Moreover, property (iv) avoids the existence of any closed surface as a subcomplex of $H^{\prime}$; hence, the edges of $H^{\prime}(\mathbf{a}, \mathbf{b})$ and $H^{\prime}(\mathbf{a}, \mathbf{c})$ cannot be faces of exactly two triangles each. Since $[\mathbf{a}, \mathbf{b}]=[\mathbf{a}, \mathbf{c}]=3 \geq(3+h) / 2$, some triangles of $H^{\prime}$ collapse from their edges " $(\mathbf{a}, \mathbf{x})$ ", where either $\mathbf{x}=\mathbf{b}$ or $\mathbf{x}=\mathbf{c}$; w.l.o.g., $\mathbf{x}=\mathbf{b}$ may be assumed.

Note that every sequence of elementary collapses of triangles from their edges “( (a, b)" or " $(\mathbf{b}, \mathbf{c})$ " produces a new complex $H^{\prime \prime}$, satisfying property (iv) (since $H^{\prime}$ collapses to $H^{\prime \prime}$ ) and property (iii), only for $\mathbf{z}=\mathbf{c}$ (since $H^{\prime \prime}(\mathbf{a}, \mathbf{c})=$ $\left.H^{\prime}(\mathbf{a}, \mathbf{c})\right)$; hence $H^{\prime \prime}$ also satisfies properties $\left(\mathrm{v}_{\mathbf{a b}}\right)$ and $\left(\mathrm{v}_{\mathbf{b c}}\right)$.

By looking in details to the three possible cases for the number $h$ of edges of $H^{\prime}(\mathbf{b}, \mathbf{c})$, we can easily obtain such a complex $H^{\prime \prime}$, with $s$ edges "(a, b)", $s$ edges " $(\mathbf{b}, \mathbf{c})$ ", and $2 s$ triangles, for $s=1,2$. By property (iv), $H^{\prime \prime}$ actually collapses to a graph.

By 5-dimensional handlebody of genus $m \geq 0$ we mean the orientable 5manifold $Y_{m}$, which admits a decomposition with exactly the 0 -handle and $m$ 1-handles. Note that $Y_{0} \cong \mathbb{D}^{5}$ (the 5-disk) and that, for $m \geq 1, \partial Y_{m} \cong$ $\#_{m}\left(\mathbb{S}^{1} \times \mathbb{S}^{3}\right)$.

Moreover, a 5-manifold with boundary $M^{5}$ is a handlebody (of genus $m$ ) iff it collapses to a graph $\Omega\left(\right.$ with $\left.\pi_{1}(\Omega) \cong \underset{m}{*} \mathbb{Z}\right)$.

Lemma 2. Let $(\Gamma, \gamma)$ be a crystallization of a closed connected orientable 5manifold $M^{5}$, and let $\varepsilon=\left(\varepsilon_{0}, \varepsilon_{1}, \ldots, \varepsilon_{5}\right)$ be a fixed permutation of $\Delta_{5}$. If there exists $\{r, s, t\} \subset \Delta_{5}$, with $s, t$ nonconsecutive in $\varepsilon_{\hat{r}}$, such that

$$
\rho_{\hat{r}}=\rho_{\hat{r} \hat{s}}+\rho_{\hat{r} \hat{t}},
$$

then $N(r, s, t)$ is a 5-dimensional handlebody $Y_{m}$ of genus $m$, with $\operatorname{rk}\left(\pi_{1}\left(M^{5}\right)\right)$ $\leq m \leq \rho$.

Proof. Set $\boldsymbol{\alpha}=\mathbf{g}_{\hat{r} \hat{s} \hat{t}}-\mathbf{g}_{\hat{r} \hat{s}} \geq 0$; this means that there are $\boldsymbol{\alpha}$ triangles in $K(r, s, t)$ more than edges in $K(r, s)$, i.e., $h(1 \leq h \leq \boldsymbol{\alpha})$ edges $\mathbf{e}_{1}, \ldots, \mathbf{e}_{h}$ of $K(r, s)$ are faces of at least two triangles of $K(r, s, t)$. Let $T_{1}, \ldots, T_{\alpha+h}$ be the $\alpha+h$ triangles of $K(r, s, t)$, which have an edge $\mathbf{e}_{i} \in K(r, s)$ as face; it is easy to check that $K(r, s, t)$ collapses to the subcomplex $\bar{K}=K(r, t) \cup K(s, t) \cup$ $\left\{\mathbf{e}_{1}, \ldots, \mathbf{e}_{h}\right\} \cup\left\{T_{1}, \ldots, T_{\alpha+h}\right\}$. By using (*) into formula (6), $\mathbf{g}_{\hat{r} \hat{s} \hat{t}}=\mathbf{g}_{\hat{r} \hat{s}}+\mathbf{g}_{\hat{r} \tilde{t}}-1$ is obtained.

Thus, the two-dimensional pseudocomplex $\bar{K}$ has: $\left[\mathbf{v}_{r}, \mathbf{v}_{t}\right]=1+\boldsymbol{\alpha},\left[\mathbf{v}_{r}, \mathbf{v}_{s}\right]=$ $h \leq \boldsymbol{\alpha},\left[\mathbf{v}_{r}, \mathbf{v}_{s}, \mathbf{v}_{t}\right]=\boldsymbol{\alpha}+h$ (for the notation, see Lemma 1a). Moreover, since $K(\Gamma)$ is a pseudocomplex triangulating a 5-manifold and having exactly six vertices, the inclusion $j: K(s, t) \rightarrow K(r, s, t)$ induces an epimorphism $j_{*}: \pi_{1}(K(r, s)) \rightarrow \pi_{1}(K(r, s, t))$ [G1, Proposition 3]. The same property holds for $\bar{K}$ and $\bar{K}(r, s)$, too, since $\bar{K}$ is obtained from $K(r, s, t)$ by elementary collapses fixing $K(r, s)$. Hence, $\bar{K}$ satisfies all the hypothesis of Lemma la and collapses to $K(s, t)$. This obviously ensures that $N(r, s, t)$ is a 5dimensional handlebody, of genus $m=\mathbf{g}_{\hat{s} \hat{t}}-1 \geq 0$. Moreover, since $s$ and $t$ are surely nonconsecutive in $\varepsilon$, [CP, Corollary 4.4] implies that $m=\mathbf{g}_{\hat{s} \hat{t}}-1 \leq$ $\rho-\max \left\{\rho_{\hat{s}}, \rho_{\hat{t}}\right\} \leq \rho$. 
The proof is now completed by recalling that the $\left(\Delta_{5}-\{s, t\}\right)$-residues of $\Gamma$, but one arbitrarily chosen, are in bijection with the generators of a suitable presentation of $\pi_{1}\left(M^{5}\right)$ [G1].

Proposition 3. Let $M^{5},(\Gamma, \gamma)$ and $\varepsilon$ be as in Lemma 2. If there exists $\{i, j\} \subset$ $\Delta_{5}$, such that $\rho_{\hat{i} \hat{j}}=0$, then either $M^{5} \cong \mathbb{S}^{5}$ or $M^{5} \cong \#_{m}\left(\left(\mathbb{S}^{1} \times \mathbb{S}^{4}\right), 1 \leq m \leq \rho\right.$. Proof. Let $\sigma=\left(\sigma_{0}, \sigma_{1}, \sigma_{2}, \sigma_{3}\right)$ be the cyclic permutation induced by $\varepsilon$ on $\Delta_{5}-\{i, j\}$. Formula (9) directly implies $\rho_{\hat{i}}=\rho_{\hat{i} \hat{\sigma}_{0}}+\rho_{\hat{i} \hat{\sigma}_{2}}\left(=\rho_{\hat{i} \hat{\sigma}_{1}}+\rho_{\hat{i} \hat{\sigma}_{3}}\right)$ and $\rho_{\hat{j}}=\rho_{\hat{j} \hat{\sigma}_{1}}+\rho_{\hat{j} \hat{\sigma}_{3}}\left(=\rho_{\hat{j} \hat{\sigma}_{0}}+\rho_{\hat{j} \hat{\sigma}_{2}}\right)$. Since $\sigma_{0}, \sigma_{2}$ (resp. $\left.\sigma_{1}, \sigma_{3}\right)$ are nonconsecutive in $\varepsilon_{\hat{i}}$ (resp. $\left.\varepsilon_{\hat{j}}\right)$, then Lemma 2 ensures that both $N\left(i, \sigma_{0}, \sigma_{2}\right)$ and $N\left(j, \sigma_{1}, \sigma_{3}\right)$ are 5-dimensional handlebodies, obviously of the same genus $m$, with $0 \leq m \leq \rho$. If $m=0$, then $M^{5} \cong \mathbb{S}^{5}$ directly follows. If $1 \leq m \leq \rho$, then $\partial N\left(i, \sigma_{0}, \sigma_{2}\right) \cong \partial N\left(j, \sigma_{1}, \sigma_{3}\right) \cong \#_{m}\left(\mathbb{S}^{1} \times \mathbb{S}^{3}\right)$; so, the result is a consequence of Corollary 4.4 and Theorem 2.1 of $[\mathrm{CH}]$.

Corollary 4. Let $M^{5},(\Gamma, \gamma)$ and $\varepsilon$ be as in Lemma 2. If there exists $i \in \Delta_{5}$, such that $\rho_{\hat{i}} \leq 2$, then either $M^{5} \cong \mathbb{S}^{5}$ or $M^{5} \cong \#_{m}\left(\mathbb{S}^{1} \times \mathbb{S}^{4}\right), 1 \leq m \leq \rho$.

Proof. As a consequence of formula (9) and Remark D, $\rho_{\hat{i}} \leq 2$ implies the existence of $j \in \Delta_{5}-\{i\}$, such that $\rho_{\hat{i} \hat{j}}=0$. Thus, the thesis follows from Proposition 3.

Lemma 5. Let $M^{5},(\Gamma, \gamma)$ and $\varepsilon$ be as in Lemma 2. If $M^{5}$ is simply connected and there exists $\{r, s, t\} \subset \Delta_{5}$, such that $N(r, s, t) \cong \mathbb{D}^{5}$, then $M^{5} \cong \mathbb{S}^{5}$.

Proof. The Mayer-Vietoris sequence of the pair $(N(r, s, t), N(\hat{r}, \hat{s}, \hat{t}))$ (with coefficients in $\mathbb{Z})$, together with Poincaré duality, easily gives $H_{4}\left(M^{5}\right)=$ $H_{3}\left(M^{5}\right)=H_{1}\left(M^{5}\right)=0$ and Tor $H_{2}\left(M^{5}\right) \cong H_{2}\left(M^{5}\right) \cong H_{2}(N(\hat{r}, \hat{s}, \hat{t}))$. On the other hand, $\mathrm{H}_{2}(N(\hat{r}, \hat{s}, \hat{t}))$ is torsion-free $(K(\hat{r}, \hat{s}, \hat{t})$ being a two-dimensional pseudocomplex); hence, $H_{2}\left(M^{5}\right)=H_{2}(N(\hat{r}, \hat{s}, \hat{t}))=0$. Now, since $H_{*}\left(M^{5}\right)=$ $H_{*}\left(\mathbb{S}^{5}\right)$, the well-known Whitehead Theorem ensures that $M^{5}$ has the same homotopy type as $\mathbb{S}^{5}$; thus, the statement follows by the Generalized Poincare Theorem.

Lemma 6. Let $M^{5},(\Gamma, \gamma)$, and $\varepsilon$ be as in Lemma 2. If there exists $\{r, s, t\} \subset$ $\Delta_{5}$, such that $N(r, s, t) \cong Y_{m}$ where $Y_{m}$ denotes a 5-dimensional handlebody of genus $m \geq 1$, then

$$
H_{*}(N(\hat{r}, \hat{s}, \hat{t}) ; \mathbb{Z}) \cong H_{*}\left(Y_{m} ; \mathbb{Z}\right) \quad \text { and } \quad H_{*}\left(M^{5} ; \mathbb{Z}\right) \cong H_{*}\left(\underset{m}{\#}\left(\mathbb{S}^{1} \times \mathbb{S}^{4}\right) ; \mathbb{Z}\right)
$$

Proof. Again, the Mayer-Vietoris sequence of the pair $(N(r, s, t), N(\hat{r}, \hat{s}, \hat{t}))$ (with coefficients in $\mathbb{Z}$ ), together with Poincaré duality easily gives: $H_{5}\left(M^{5}\right) \cong$ $\mathbb{Z}, H_{4}\left(M^{5}\right) \cong H_{1}\left(M^{5}\right) \cong \bigoplus_{m} \mathbb{Z}, H_{3}\left(M^{5}\right)=0$, and Tor $H_{2}\left(M^{5}\right) \cong H_{2}\left(M^{5}\right) \cong$ $H_{2}(N(\hat{r}, \hat{s}, \hat{t}))$. As in the proof of the preceding lemma, $H_{2}(N(\hat{r}, \hat{s}, \hat{t}))$ is torsion-free; hence, $H_{2}\left(M^{5}\right)=H_{2}(N(\hat{r}, \hat{s}, \hat{t}))=0$. Finally, $H_{1}(N(\hat{r}, \hat{s}, \hat{t}))$ is computed by means of the splitting sequence

$$
0 \rightarrow \bigoplus_{m} \mathbb{Z} \rightarrow H_{1}(N(\hat{r}, \hat{s}, \hat{t})) \oplus\left(\bigoplus_{m} \mathbb{Z}\right) \rightarrow \bigoplus_{m} \mathbb{Z} \rightarrow 0 .
$$

Proposition 7. Let $(\Gamma, \gamma)$ be a crystallization of a closed connected orientable 5-manifold $M^{5}$. If there exists a cyclic permutation $\varepsilon$ of $\Delta_{5}$, such that $\rho_{\varepsilon}(\Gamma)=$ $\rho \leq 7$, then either $M^{5} \cong \mathbb{S}^{5}$ or $M^{5} \cong \#_{m}\left(\mathbb{S}^{1} \times \mathbb{S}^{4}\right), 1 \leq m \leq \rho$. 
Proof. Set $\rho^{* *}=\min \left\{\rho_{\hat{i} j} \mid i, j \in \Delta_{5}, i \neq j\right\}$. By Proposition 3, the case $\rho^{* *}=0$ directly implies the thesis; hence, $\rho^{* *} \geq 1$ may be supposed. Let us further set $\rho^{*}=\min \left\{\rho_{\hat{i}} \mid i \in \Delta_{5}\right\}$. By Corollary $4, \rho^{*} \geq 3$ may also be supposed. Now, formulae (7) and (8) imply that, if $\rho(\Gamma) \leq 7$, then $\rho^{*} \leq 4$ (since $\mathbf{g}_{\mathscr{B}} \geq 1$, for every $\mathscr{B} \subseteq \Delta_{5}$ ). If $r \in \Delta_{5}$ is such that $\rho_{\hat{r}}=\rho^{*}$, then the only possible cases for formula (9) are:

(a) $\rho_{\hat{r}}=3=(1+1+1+1+2) / 2$;

(b) $\rho_{\hat{r}}=4=(1+1+2+2+2) / 2$;

(c) $\rho_{\hat{r}}=4=(1+1+1+2+3) / 2$.

Note that the further case $\rho_{\hat{r}}=4=(1+1+1+1+4) / 2$ cannot hold by Remark C.

Anyway, in all the previous three cases (a), (b), (c), there exist $s, t$ nonconsecutive in $\varepsilon_{\hat{r}}$ such that $\rho_{\hat{r}}=\rho_{\hat{r} \hat{s}}+\rho_{\hat{r} \hat{t}}$; thus, Lemma 2 implies that $N(r, s, t)$ is homeomorphic to a 5-dimensional handlebody $Y_{m}$ of genus $m$, with $\operatorname{rk}\left(\pi_{1}\left(M^{5}\right)\right) \leq m \leq \rho$.

If $m=0$, i.e., if $N(r, s, t) \cong \mathbb{D}^{5}$, then $M^{5}$ is simply connected and Lemma 5 concludes the proof. Let us assume $N(r, s, t) \cong Y_{m}, 1 \leq m \leq \rho$. Set $\{u, v, w\}=\Delta_{5}-\{r, s, t\}$; by the hypothesis on $\{r, s, t\}, u$ may be supposed to be nonconsecutive both to $v$ and to $w$ (in $\varepsilon$ ). If $\rho_{\hat{v}}=\rho_{\hat{u} \hat{v}}+\rho_{\hat{v} \hat{w}}$ (or $\left.\rho_{\hat{w}}=\rho_{\hat{u} \hat{w}}+\rho_{\hat{v} \hat{w}}\right)$, then the thesis directly follows from Lemma 2 by making use of Corollary 4.4 and Theorem 2.1 of $[\mathrm{CH}]$.

Let us assume

$$
\rho_{\hat{v}}-\rho_{\hat{u} \hat{v}}-\rho_{\hat{v} \hat{w}} \geq 1 \quad \text { and } \quad \rho_{\hat{w}}-\rho_{\hat{u} \hat{w}}-\rho_{\hat{v} \hat{w}} \geq 1 .
$$

Formula (6) gives

$$
\begin{aligned}
& \mathbf{g}_{\hat{u} \hat{v} \hat{w}}=\mathbf{g}_{\hat{u} \hat{v}}+\mathbf{g}_{\hat{v} \hat{w}}-1+\left(\rho_{\hat{v}}-\rho_{\hat{u} \hat{v}}-\rho_{\hat{v} \hat{w}}\right) \geq \mathbf{g}_{\hat{u} \hat{v}}+\mathbf{g}_{\hat{v} \hat{w}}, \\
& \mathbf{g}_{\hat{u} \hat{v} \hat{w}}=\mathbf{g}_{\hat{u} \hat{w}}+\mathbf{g}_{\hat{v} \hat{w}}-1+\left(\rho_{\hat{w}}-\rho_{\hat{u} \hat{w}}-\rho_{\hat{v} \hat{w}}\right) \geq \mathbf{g}_{\hat{u} \hat{w}}+\mathbf{g}_{\hat{v} \hat{w}}
\end{aligned} ;
$$

i.e.,

(+) $\quad \mathbf{g}_{\hat{u} \hat{w}} \leq \mathbf{g}_{\hat{u} \hat{v} \hat{w}}-\mathbf{g}_{\hat{v} \hat{w}} \quad$ and $\quad \mathbf{g}_{\hat{u} \hat{w}} \leq \mathbf{g}_{\hat{u} \hat{v} \hat{w}}-\mathbf{g}_{\hat{v} \hat{w}}$.

By Lemma $6, \chi(K(u, v, w))=1-m \leq 0$; on the other hand,

$$
\chi(K(u, v, w))=3-\left(\mathbf{g}_{\hat{u} \hat{w}}+\mathbf{g}_{\hat{u} \hat{w}}+\mathbf{g}_{\hat{v} \hat{w}}\right)+\mathbf{g}_{\hat{u} \hat{v} \hat{w}} .
$$

Hence, $0 \leq \mathbf{g}_{\hat{u} \hat{v} \hat{w}}-\mathbf{g}_{\hat{v} \hat{w}} \leq \mathbf{g}_{\hat{u} \hat{v}}+\mathbf{g}_{\hat{u} \hat{w}}-3$, and, by $(+), \mathbf{g}_{\hat{u} \hat{v}} \geq 3$, $\mathbf{g}_{\hat{u} \hat{w}} \geq 3$.

Moreover, by making use of $\rho \leq 7, \rho^{* *} \geq 1$, and relations (\$), we obtain from formula (5)

$$
\begin{aligned}
& \mathbf{g}_{\hat{u} \hat{v}}=1+\rho-\rho_{\hat{u}}-\left(\rho_{\hat{v}}-\rho_{\hat{u} \hat{v}}\right) \leq 3, \\
& \mathbf{g}_{\hat{u} \hat{w}}=1+\rho-\rho_{\hat{u}}-\left(\rho_{\hat{w}}-\rho_{\hat{u} \hat{w}}\right) \leq 3 .
\end{aligned}
$$

Hence, $\mathbf{g}_{\hat{u} \hat{v}}=\mathbf{g}_{\hat{u} \hat{w}}=3$ and $\mathbf{g}_{\hat{u} \hat{v} \hat{w}}=\mathbf{g}_{\hat{v} \hat{w}}+3$. This means that the twodimensional complex $K(u, v, w)$ satisfies the hypotheses (i) and (ii) of Lemma $1 \mathrm{~b}$; moreover, hypothesis (iii) and (iv) hold for [G1, Proposition 3] and by Lemma 6 respectively. As a consequence, $K(u, v, w)$ collapses to a graph, and therefore $N(u, v, w)=N(\hat{r}, \hat{s}, \hat{t}) \cong Y_{n}$. Since $N(r, s, t) \cong Y_{m}$ is assumed, $m=n$ obviously holds; the thesis again follows from Corollary 4.4 and Theorem 2.1 of $[\mathrm{CH}]$. 
Proof of the main theorem and of Corollary $\mathrm{I}$. The proof of the main theorem is a direct consequence of Proposition 7. On the other hand, the genus of $\mathbb{S}^{3} \times \mathbb{S}^{2}$ is proved to be $\leq 8$ in [GG]. Hence Corollary I again follows from Proposition 7.

\section{REFERENCES}

[B] D. Barden, Simply connected five-manifolds, Ann. of Math. (2) 82 (1965), 365-385.

[BM] J. Bracho and L. Montejano, The combinatorics of colored triangulations of manifolds, Geom. Dedicata 22 (1987), 303-328.

[C] M. R. Casali, A combinatorial characterization of 4-dimensional handlebodies, Forum Math. 4 (1992), 123-134.

[CH] A. Cavicchioli and F. Hegenbarth, On the determination of PL-manifolds by handles of lower dimension, Topology Appl. (to appear).

[CP] R. Chiavacci and G. Pareschi, Some bounds for the regular genus of closed PL manifolds, Discrete Math. 82 (1990), 165-180.

[FG] M. Ferri and C. Gagliardi, The only genus zero n-manifold in $\mathbb{S}^{n}$, Proc. Amer. Math. Soc. 85 (1982), 638-642.

[FGG] M. Ferri, C. Gagliardi, and L. Grasselli, A graph-theoretical representation of PL manifolds-A survey on crystallizations, Aequationes Math. 31 (1986), 121-141.

[G1] C. Gagliardi, How to deduce the fundamental group of a closed n-manifold from a contracted triangulation, J. Combin. Inform. System Sci. 4 (1979), 237-252.

[G2] Extending the concept of genus to dimension n, Proc. Amer. Math. Soc. 81 (1981), 473-481.

[G3] _- Surface maps and n-dimensional manifolds, Atti IV Convegno di Topologia, Sorrento (Na)-September 1988, Rend. Circ. Mat. Palermo 24 (1990), 97-126.

[GG] C. Gagliardi and L. Grasselli, Representing products of polyhedra by products of edge-coloured graphs, J. Graph Theory (to appear).

[Gl] L. C. Glaser, Geometrical combinatorial topology, Van Nostrand Reinhold Math. Stud., Van Nostrand Reinhold, New York, 1970.

[HW] P. J. Hilton and S. Wylie, An introduction to algebraic topology-Homology theory, Cambridge Univ. Press, Cambridge, 1960.

[LM] S. Lins and A. Mandel, Graph-encoded 3-manifolds, Discrete Math. 57 (1985), 261-284.

[P1] M. Pezzana, Sulla struttura topologica delle varietá compatte, Atti Sem. Mat. Fis. Univ. Modena 23 (1975), 269-277.

[P2] Diagrammi di Heegaard e triangolazione contratta, Boll. Un. Mat. Ital. 12 (1975), 98-105.

[RS] C. Rourke and B. Sanderson, Introduction to piecewise-linear topology, Springer Verlag, New York and Heidelberg, 1972.

[S] S. Smale, On the structure of 5-manifolds, Ann. of Math. (2) 82 (1962), 38-46.

[V] A. Vince, n-graphs, Discrete Math. 72 (1988), 367-380.

[W] A. T. White, Graphs, groups and surfaces, North-Holland, Amsterdam, 1973.

Dipartimento di Matematica, via Campi 213/B, I-41100 Modena, Italia

E-mail address: GAGLIARDIQIMOVX2.UNIMO.IT 